\title{
Effect of Numerous Data Sets on Performance Prediction
}

\author{
Jyoti Upadhyay \\ Research Scholar \\ AISECT University \\ Bhopal, M.P.
}

\author{
Pratima Gautam, PhD \\ Dean IT Dept. \\ AISECT University \\ Bhopal, M.P.
}

\begin{abstract}
There are many factors, which may affects performance. But the number of factors also affects on result of computational model. We are presenting a computational model to forecast students' performance. To calculate we will use 8 different factors that are directly or indirectly influence performance. Influencing factors how much correlated to each other we also present this. Through this paper we classify those factors using fuzzy decision tree.
\end{abstract}

\section{Keywords}

Data Mining, Fuzzy Decision Tree, Classification, correlation and Prediction

\section{INTRODUCTION}

\subsection{Educational Data Mining}

Data mining is a knowledge mining technique. We may refer it with knowledge discovery process. Data mining provides different techniques to mine information from the database. If user wants to mine information from educational data from then it becomes Educational Data Mining (EDM)

According to www.educationalmining.com_"Educational

Data Mining is an emerging discipline, concerned with developing methods for exploring the unique types of data that come from educational settings, and using those methods to better understand students, and the settings which they learn".

We have lots of data mining algorithms and techniques to analyze data. Some techniques are Classification, Clustering, Regression, Artificial intelligence, Neural networks, Association Rules, Decision Trees, Genetic Algorithm, Nearest Neighbor method etc., are used for knowledge discovery from databases. For our work we will use classification technique.

\subsection{Classification}

Most commonly data-mining techniques are Classification. Classification is used to develop a model that can classify the data set. A classification technique commonly works through decision tree or neural network-based classification algorithms. Data classification process involves mainly two steps learning and classification. In Learning step the training set are analyzed by classification algorithm [1]. After applying model test data are used to estimate the accuracy of the classification rules. If the accuracy is acceptable the rules can be applied to the new data tuples. For our work we are going to work upon students' data to predict success possibility in college exam and factors, which may affect students' performance. Some classification models are:
- Decision tree induction

- Bayesian Classification

- Neural Networks

- Classification Based on Association

\subsection{Decision tree}

A decision tree is a classification technique, which classifies data set into possible classes. Decision trees are used to extract knowledge by making decision rules from the large amount of available information [2]. In decision tree each leaf node represents a decision and branch node represents a choice of alternatives attributes. From root node, users split each node recursively according to decision tree learning algorithm. The final result of decision tree is a possible scenario of decision and its result.

In our paper we will take fuzzy decision tree. Fuzzy decision trees grow in a top-down way. It uses recursively partitioning the training data into segments with similar outputs.

To construct Fuzzy decision tree apply fuzzy sets to describe (quantify) attributes and then use the ID3 approach. Fuzzy entropy, information gains or gain ratio are used as a measure of attribute selection [8].

\section{METHODOLOGY}

\subsection{Data Collection}

For data collection we select an educational organization We collect 130 data according to our need. For experiment we will take following parameter.

Table 1: Parameters

\begin{tabular}{|c|l|l|}
\hline S. No & Parameter & Values \\
\hline 1 & Cast Category & SC, ST, OBC, GEN \\
\hline 2 & Previous Percent & Percentage of $12^{\text {th }}$ \\
\hline 3 & $\begin{array}{l}\text { Attendance } \\
\text { percent }\end{array}$ & Attendance Percentage \\
\hline 4 & Location & $\begin{array}{l}\text { Rural,SemiUrban, } \\
\text { Urban }\end{array}$ \\
\hline 5 & Sports interest & Avg, Good, Poor \\
\hline 6 & Parents income & Avg, Good, Poor \\
\hline 7 & $\begin{array}{l}\text { Unittest } \\
\text { Performance }\end{array}$ & Avg, Good, Poor \\
\hline 8 & Recent Result & Fail, Pass \\
\hline
\end{tabular}

Above Parameter list Recent Result is label and rest are decision parameter. 


\subsection{Process}

We filter missing data using excel feature. We have to transform data set into fuzzy data set. Fuzzy set theory is an approach to represent uncertainty. A fuzzy set A is characterized by its membership function (MF). MF range is belonging to the unit interval. MF represents the degree of membership of the point in the set A [3]. We transformed data set into fuzzy set. Transformed data set shows in table 2.

Table 2Transformed Data

\begin{tabular}{|c|c|c|c|c|c|c|c|c|}
\hline Ninase of stutentis & Cast & Location & reven r rsilt & Funzy atn & fuzxy prev resalt & spons Ineresst & $t$ perents manse & Uniti test Peff \\
\hline Sunet Singh & $\mathrm{GEN}$ & Uthan & Fail & gond & thierd & avg & ach & porx \\
\hline Sweta Prasad & ST & Semi-rbara & Fail & good & seeond & good & sod & h \\
\hline Neclam Singh & GEN & Uthan & Pass & good & scoond & poor & iod & $\cos$ \\
\hline B.Mallur Kumar & $\mathrm{GEN}$ & Rural & & AVG & thierd & poor & max & ${ }_{p x p}^{p x p}$ \\
\hline KaranAura & $\mathrm{GEN}$ & Utran & Pass & good & second & good & ood & $\cos 0$ \\
\hline Alkankhha Singh & GEN & Utran & Fail & AVG & seeond & poor & ood & pox \\
\hline Garary Gyali & $\mathrm{GEN}$ & Semi-rrban & Pass & gond & seeond & good & aE. & gotod \\
\hline Sthainy Joseph & $\mathrm{GEN}$ & Utroan & & good & first & good & pos & $\operatorname{sos} x$ \\
\hline Kamal Karayan & SC & Rural & Fal & good & seovnd & good & ab. & pox \\
\hline
\end{tabular}

After Transformation we apply filtration on data set. Then calculate weight of each attribute. We depicted it on Figure 1. It shows that Unit test performance and attendance has high weight comparing to other. Cast has lowest weight. We use Chi square test to calculating weight.

\begin{tabular}{|c|c|}
\hline & weight \\
\hline 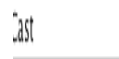 & 1.725 \\
\hline iports minest & Ssllj \\
\hline Wuzzy peryeselt & 13261 \\
\hline Jarents mowe & 13692 \\
\hline .odation & 18379 \\
\hline Intitestpert & 42882 \\
\hline ivzy ith & 453.32 \\
\hline
\end{tabular}

Figure 1: weight of attribute

After Calculating weight apply validation and CHAID to construct Decision tree. The condensation of CHAID is Chi-squared Automatic Interaction Detector. It is tree classification methods originally proposed by Kass (1980). It has been developed for categorical variables [ 5].

CHAID will "build" non-binary trees (i.e., trees where more than two branches can attach to a single root or node), based on a relatively simple algorithm that is particularly well suited for the analysis of larger datasets. CHAID algorithm will often effectively yield many multiway frequency tables (e.g., when classifying a categorical response variable with many categories, based on categorical predictors with many classes).

CHAID is a technique that recursively splits a data set into discrete segments. These are nodes. Nodes are split in such a way that the difference of the resultant variable is minimized [6].

To construct Decision tree we have to select root node from the list of decision attributes. Attribute selection has been performed by information gain. Basically information gain can be defined by

Information gain = Information before splitting Information after splitting.

Information gain is influenced about choosing attributes with a large number of values. This may result in over fitting. So that modifications in information gain is Gain ratio. Gain ratio takes number and size of branches into account when choosing a decision attribute. It corrects the information gain by taking the intrinsic information of a split into account [4].

\subsection{Tools}

For our work we selected Rapid miner tool. Rapid Miner is an open source to maximize the value of predictive analytics. Through Rapid Miner we can makes predictive analytics lightning-fast, drastically reducing the time to discover risks. It provides powerful visual design evaluation speeds repetitive tasks, considerably reducing the time that spent on data access and preparation. Rapid miner helps connect to any data at any scale and desirable Optimization schemes [7].

\section{OUTCOMES}

Generated decision tree shows in Figure 2. We can See from Figure 2 and Figure 3 that if Attendance of a candidate Average then most probable performance will be poor specially when candidate from rural or semi urban area. If Attendance is good then performance value will be increases. Figure 3 shows fuzzy performance rule, extract from decision tree. All parameters are not plays vital role in performance prediction it can be easily determine by decision tree.

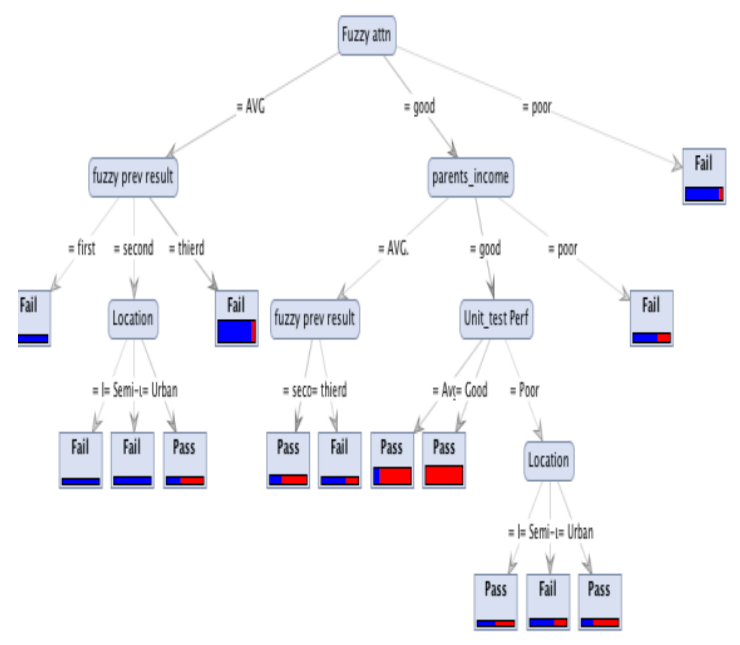

Figure 2 fuzzy decision trees 


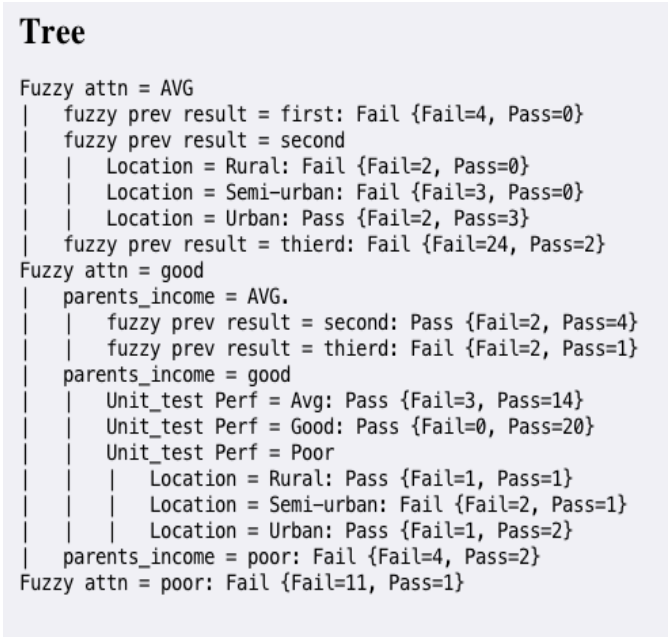

Figure 3: Decision rule

In Figure 4 shows predicted result and confidence value of performance. We categorized performance in fail and pass so that we can see in first row that original and predicted result is same so that confidence of fail is greater than confidence of pass.

\begin{tabular}{|c|c|c|c|c|c|c|c|c|}
\hline & & & & & Filter $(112 / 11$ & 2 exampless: : a & & \\
\hline Row No. & recent result & tpredicitionfrece.... & confidencefefail) & confidencepeass) & Nmze of st... Cast & Location & Fuzzy attin & fuzzy pre \\
\hline 1 & Fail & Fail & 0.667 & 0.333 & Sumeet Sing CEN & Urban & good & thierd \\
\hline 2 & Fail & Pass & 0.176 & 0.824 & Sweta Prasa ST & Semi-urban 9 & good & second \\
\hline 3 & Pass & Pass & 0 & 1 & Neelam Sinç CEN & Urban & good & second \\
\hline 4 & Pass & Pass & 0 & 1 & Karan Arora CEN & Urban & good & second \\
\hline 5 & Fail & Pass & 0.400 & 0.600 & Akanksha SI CEN & Urban & AVC & second \\
\hline 6 & Pass & Pass & 0.333 & 0.667 & Gaurav Gay GEN & Semi-urban 9 & good & second \\
\hline 7 & Fail & Pass & 0.333 & 0.667 & Kamal Nara SC & Rural & good & second \\
\hline 8 & Pass & Pass & 0.333 & 0.667 & Avind Tanc SC & Urban & good & thierd \\
\hline 9 & Pass & Pass & 0.333 & 0.667 & Shweta Sing CEN & Urban & good & first \\
\hline 10 & Pass & Pass & 0 & 1 & Deepal Pat CEN & Urban & good & second \\
\hline 11 & Fail & fail & 0.923 & 0.077 & Vijay Phulvic CEN & Semi-urban A & AVC & thierd \\
\hline 12 & Fail & Fail & 0.917 & 0.083 & C.Megha CEN & Rural & poor & thierd \\
\hline 13 & Fall & Fall & 0.917 & 0.083 & Ramneek Bi SC & Rural & poor & thierd \\
\hline 14 & Pass & Pass & 0.176 & 0.824 & D.Sneha SC & Urban & good & second \\
\hline 15 & fail & Fail & 0.923 & 0.077 & C.Mahesh K GEN & Urban & AVC & thierd \\
\hline 16 & Pass & Pass & 0.500 & 0.500 & Shubham SC SC & Rural & good & thierd \\
\hline 17 & Fail & Fail & 1 & 0 & Prashant Ku SC & Semi-urban $A$ & AVC & second \\
\hline 18 & Fall & Fail & 0.917 & 0.083 & Dhriti Pande CEN & Urban & poor & thierd \\
\hline 19 & Fail & Fail & 0.917 & 0.083 & Sanju Tandi SC & Rural & poor & thierd \\
\hline $20=-2+2$ & Fail & Fail & 1 & 11 & Latinter Sin C.FN & Gemiluman A $A$ & avr. & cernont \\
\hline
\end{tabular}

\section{Figure 4 Predicted result and Confidence Value}

Figure 5 shows Performance Vector, which shows accuracy of model is $99 \%$ and classification error is $0.89 \%$.

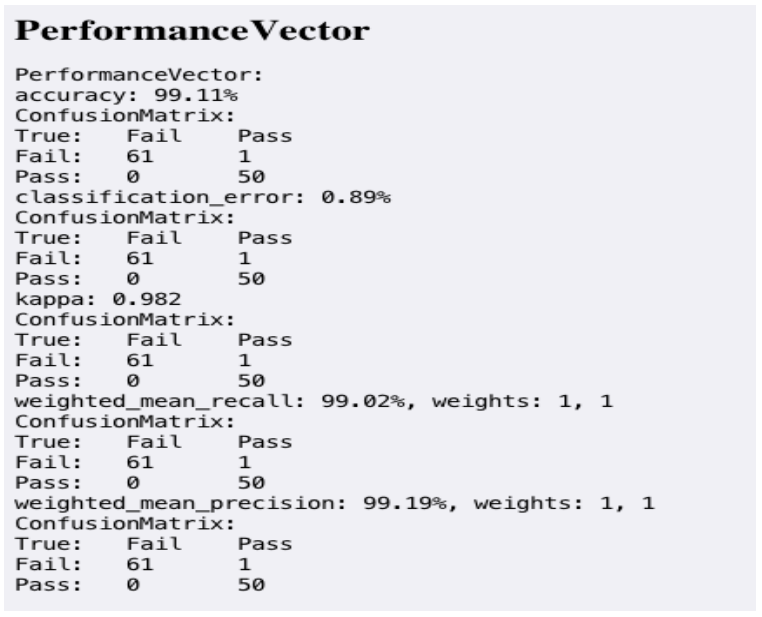

Figure 5: Performance Vector

\section{CONCLUSION}

And Fuzzy Decision rule will be extract like in Figure 3 Decision tree shows that attendance is play an important role in students' performance. Students' performance may affect by living location and parents income also. Cast has no role in performance. Organization, Teachers and students also can take advantage from this model. They can find out factor which may affect result and do work on it. Different factors may help to predict performance. If through a factor it is difficult to predict performance then other factor my help for prediction. We perform this task for 150 samples. After filtration we got 112 samples in which only 1 produce wrong prediction.

\section{REFERENCES}

[1] Ramageri, Mrs. Bharati M. "DATA MINING TECHNIQUES AND APPLICATIONS ." Indian Journal of Computer Science and Engineering Vol. 1 No. 4 301-305 (2010): 301-305.

[2] Sonia Joseph, Laya Devadas. "Student's Performance Prediction Using Weighted Modified ID3 Algorithm ." International Journal of Scientific Research Engineering \& Technology (IJSRET), 4.5 (2015): 571.

[3] Zadeh, L. A. "fuzzy set." information and control 8 (1965): 338-352.

[4] Alec Pawling, Nitesh V. Chawla, and Amitabh Chaudhary. "Computing Information Gain in Data Streams." TDM 2005: 2005 Temporal Data Mining Workshop (2005): 72-81.

[5] G. V. Kass, "An Exploratory Technique for Investigating Large Quantities of Categorical Data", Applied Statistic, Vol. 29, (1980): 119-127.

[6] M. Ramaswami , R. Bhaskaran , "A CHAID Based Performance Prediction Model in Educational Data Mining",International Journal of Computer Science Issues, Vol. 7, (2010) :10-18

[7] https://rapidminer.com/products/studio/, dated march 2016.

[8] Xiaodong Liu,Xinghua Fenga, andWitold Pedryczc, "Extraction of fuzzy rules from fuzzy decision trees: An axiomatic fuzzy sets (AFS) approach", Data \& Knowledge EngineeringVolume 84, (2013) , 1-25 